\title{
Psychometric properties of the Farsi version of Attitudes to Aging Questionnaire in Iranian older adults
}

This article was published in the following Dove Press journal:

Clinical Interventions in Aging

22 September 2017

Number of times this article has been viewed

\author{
Nahid Rejeh' \\ Majideh Heravi-Karimooi' \\ Mojtaba Vaismoradi² \\ Pauline Griffiths ${ }^{3}$ \\ Maryam Nikkhah ${ }^{4}$ \\ Tahereh Bahrami ${ }^{4}$ \\ 'Elderly Care Research Centre, \\ Faculty of Nursing and Midwifery, \\ Shahed University, Tehran, Iran; \\ ${ }^{2}$ Faculty of Nursing and Health \\ Sciences, Nord University, Bodø, \\ Norway; ${ }^{3}$ College of Human and \\ Health Sciences, Swansea University, \\ Swansea, UK; ; ${ }^{4}$ aculty of Nursing \\ and Midwifery, Shahed University, \\ Tehran, Iran
}

Background: Attitudes to the aging process are affected by the individual's sociocultural background. The measurement of this important concept among older people in various societies requires the use of tools that are able to demonstrate both reliability and validity.

Objective: The objectives of this study were to translate and validate the Attitudes to Aging Questionnaire (AAQ) and investigate its psychometric features among Iranian older people.

Methods: In this methodological study, the Farsi version of the AAQ was validated among 400 Iranian older adults who were members of citizen clubs in an urban area of Iran. Content, face and construct (exploratory factor analysis) validities of the AAQ were assessed. In addition, its reliability was assessed in terms of internal consistency and stability. For discriminant validity, the discriminant power of the AAQ in terms of gender and education levels was evaluated. Criterion validity showed a significant correlation between the most subscales of the AAQ and the Short Form 36 (SF-36) and World Health Organization Quality of Life (WHOQOL) questionnaires. Descriptive and inferential statistics were used for data analysis.

Results: The exploratory factor analysis confirmed the construct validity of the AAQ. The result of the test-retest reliability with a 2-week interval was satisfactory and reported as $r=0.90$ $(p<0.001)$. The Cronbach's alpha coefficient was reported as 0.75 for the whole instrument and $0.85-0.93$ for its dimensions. There were associations between the AAQ subscales, SF-36 quality of life $(r=0.56, p<0.001)$ and WHOQOL-BREF $(r=0.78, p<0.001)$, indicating an appropriate convergent validity. The ability to discriminate between male and female participants and those with lower and higher education levels was demonstrated, thus lending support to claims for adequate known-group validity.

Conclusion: The Farsi version of the AAQ showed favorable validity and reliability. Therefore, it can be used for assessing attitudes toward aging among older adults.

Keywords: aging, Attitudes to Aging Questionnaire, older adults, psychometrics properties, reliability, validity, elderly

\section{Introduction}

The growth rate of the older adult population in all societies now exceeds that of the total population. In 1950, it was estimated that $9.2 \%$ of the world's population were at least 60 years old; this has risen to $11.7 \%$ in 2013 and is expected to reach $21.1 \%$ by $2050 .{ }^{1}$ It is estimated that older people will soon constitute $60 \%$ of the customers of the health care system in Iran. ${ }^{2}$ According to an Iranian population and housing census published in $2010, \sim 8.2 \%$ of the total population of Iran and $6.1 \%$ of the population aged $\geq 60$ years were living in Tehran, the capital of Iran. ${ }^{3}$ 
The old-age phase of the human life span is affected by interpersonal relationships and cultural context, especially attitudes toward the older person and aging, as well as physical, psychological and social aspects. ${ }^{4}$ To learn about older people's health, well-being, personal identity and the consequences of individuals' behaviors, the ability to understand the experiences and attitudes of older people is essential. ${ }^{5}$ The process of aging can be regarded as problematic if there is a cultural overemphasis on declining health and well-being and an expectation of physical and cognitive disabilities. Such negative stereotypes allude to the concept of "ageism" indicating negative attitudes toward aging. ${ }^{6}$ While aging is described as a debilitating process characterized by an accumulation of functional limitations due to gradual physical and/or cognitive decline, it is not always experienced as such by older people. On the contrary, a majority of older people have positive perceptions of aging and/or do not even label themselves as "old". ' According to Levy's stereotype embodiment theory, prolonged exposure to negative aging stereotypes during life can result in the development of negative self-stereotypes that are activated as the individual reaches old age themselves. It has been shown that people with more negative age stereotypes showed significant worse memory performance over 38 years than those with less negative age stereotypes. ${ }^{8}$ Likewise, those who held negative age stereotypes were more likely to experience a cardiovascular event in the following 38 years than those with more positive age stereotypes. These studies indicate that as individuals grow older, negative age stereotypes become self-fulfilling. Aging stereotypes internalized from society and culture are social constructs that affect the individual's attitudes toward their own aging when, in time, they perceive themselves as an older person. ${ }^{9}$ People's attitudes about aging can affect physical and psychological conditions in old age and can be a strong predictor of health and mortality. ${ }^{10}$ Attitudes toward one's own aging include the cognitive, affective and assessment components of behaviors regarding the process of aging as a personal experience. ${ }^{11}$ Aging is a process rather than a state and is a particular personal experience; therefore, an individual's attitude toward the aging process can affect quality of life (QoL) in later years. ${ }^{12}$ A positive attitude to aging leads to higher QoL, greater satisfaction with life and less depression and loneliness. ${ }^{13}$ In addition, attitudes toward aging may be influenced by the older person's ability to undertake activities of daily living (ADLs). ${ }^{12}$ Negative attitudes toward one's own age evaluated by the Attitudes to Aging Questionnaire (AAQ) are positively associated with a lower QoL. ${ }^{14-16}$
Understanding attitudes toward aging and the behaviors of older people in the process of aging enable health care providers to take effective measures, prevent potential healthrelated issues and improve QoL. ${ }^{17-19}$

Searching of databases such as the PubMed (including MEDLINE) and CINAHL demonstrated that many studies used the Philadelphia Geriatric Center Morale Scale (PGCMS) to assess dimensions of emotional adjustments in individuals aged 70-90 years. The PGCMS has been designed to provide a measure of morale and psychological well-being for use in social studies of gerontology. The PGCMS consists of 15 items that capture a very restricted snapshot of the aging experience. This tool has been used in a variety of gerontological studies; it cannot however flexibly and comprehensively measure attitudes toward aging. ${ }^{20}$ Studies of older people indicate that older adults with negative attitudes at baseline describe themselves as having worse physical health over a 28 -year period and live an average of 7.5 years shorter than those with more positive attitudes. ${ }^{21,22}$ In addition, older adults with a negative opinion of old age are less likely to have involved themselves in good health care behaviors during the 20 years before their own old age. ${ }^{23}$ Therefore, age stereotypes held by older adults and their attitudes toward their own aging process should be taken into account, rather than solely the objective parameters of physical or psychological distress. Awareness (and review) of one's own attitudes toward old age can influence aging positively. ${ }^{24}$ Subjective aging is a superordinate construct including various related concepts reflecting the ways in which individuals experience the aging process. ${ }^{25}$ Despite a growing interest in the personal experiences of and attitudes to the aging process, there is a paucity of instruments able to recognize and study such indicators. This deficit then results in a lack of information about older adults' own attitudes toward aging. ${ }^{26} \mathrm{~A}$ commonly used measure of subjective aging is the Attitude Toward Own Aging (ATOA) tool that contained five statements to assess the general attitude of the participant toward aging. It has been argued however that the ATOA is inadequate as a general instrument to flexibly and completely evaluate attitudes toward one's own aging. ${ }^{27}$

To counter such arguments, there is a need to develop an adaptation of the World Health Organization Quality of Life (WHOQOL) group assessment tool and the AAQ to evaluate, flexibly and completely, the aging process as an individual experience from the perspective of older people. While the AAQ and ATOA take into account losses and gains experienced over the life course, the AAQ conceptualization of older people's attitudes toward their own aging is multidimensional 
and can be used in cross-cultural settings. Both instruments have been used in several countries among older people, at times with some adjustment to their content.

Older adults are the only group in society who are qualified to comment on the experience of aging as they have the most intimate knowledge of coping during the aging process. In this respect, the AAQ can capture the personal experiences of aging in the context of more general attributes about attitudes to aging. ${ }^{28}$

The AAQ focuses primarily on three different dimensions of aging. The first dimension focuses on psychosocial losses and consists of statements related to negative attitudes toward old age. For older people who experience psychosocial losses, old age is perceived as a negative experience, incorporating social and psychological losses (eg, "as I get older I find it more difficult to make new friends"). The second subscale considers physical functioning in terms of health and exercise and the experience of aging itself. The third subscale entitled "psychological growth" demonstrates that aging can be understood as a time associated with wisdom and growth. The longevity development perspective on aging is present in this subscale, including "wisdom comes with age", which also highlights gains for the individual and others such as "I want to give a good example to younger people". The AAQ utilizes both general and experiential approaches to aging. For instance, the general approach contains statements such as the "old age is a time of loneliness" and experiential statements such as "I see old age mainly as a time of loss". The questionnaire's instructions specify that individuals need to report how they feel about growing older with regard to their life in general.

The psychometric properties of the AAQ have been examined in an international dataset. ${ }^{14}$ Psychometric analysis of the AAQ has also been performed in Spain, Brazil, Scotland, Norway and English Canadian populations. ${ }^{26,28-30}$ It was found that the AAQ had appropriate validity and reliability. When older people had positive attitudes toward their own aging process, they are more likely to experience good health and their QoL was improved. ${ }^{28}$ Participants with more positive attitudes also showed less depressive symptoms. A gender influence on attitudes toward one's own aging process has not been evidenced; however, higher educated older adults demonstrate more positive attitudes than those without such education. ${ }^{28,29}$ However, according to the statistical analysis for confirming the subscales of the AAQ, the factor structure of the AAQ was not as obvious as one might expect. ${ }^{26,27,29,30}$

The AAQ has demonstrated a suitable psychometric performance, acceptable Cronbach's alpha coefficients, high test-retest reliability, discriminant validity and concurrent validity in different contexts and cultures. ${ }^{27}$ However, no standard Farsi version of this questionnaire is available. The adaptation of this tool for use in Iran would enable its use in studies on aging, older people's status and the effects of health care and social interventions. Therefore, the purpose of this study was to evaluate the psychometric properties of the Farsi version of the AAQ. The objectives of the current study were to examine the following psychometric properties of the scale: 1) exploratory factor analysis; 2) known-group differences; 3) correlations with the Short Form 36 (SF-36) and WHOQOL-BREF and 4) internal consistency with item analysis.

\section{Background of the AAQ}

There is no standard method for the assessment of attitudes toward aging, and there is a lack of information about older adults' own attitudes toward it. The AAQ has been developed using an appropriate method of instrument development collated by the WHOQOL in the context of WHOQOL measures for the use with older adults. ${ }^{31}$ It was developed following intense theoretical debate among international experts followed up with focus groups with older adults to confirm or adjust the questionnaire items. ${ }^{28}$ The AAQ was developed as one part of a larger 3-year international project on QoL among older people in collaboration with the World Health Organization (WHO) and was funded by the European Commission 5th Framework Programme. ${ }^{32,33}$ The study involved a partnership of 23 centers representing a wide range of cultures, including Europe, North America, Japan, China and Turkey. In taking a lifespan development approach, the experience of older age is conceptualized as multidimensional concept and one wherein older adults experience both losses and gains. ${ }^{31}$ The main aims of the project were to develop a measure to assess QoL in older adults and to then assess QoL in an innovative cross-cultural study of healthy older adults. It was hypothesized that attitudes to aging was important to aging and QoL. Therefore, the concurrent development of the AAQ in this study is considered as one part of this large project.

\section{Methods \\ Study design}

This was a descriptive, methodological study.

\section{Setting and participants}

The study sample was 400 older men and women. These participants were living in their own homes or with their own 
children/relatives and were also members of citizen clubs in Tehran, Iran. More than 60,000 older people attend home health care centers in the 22 districts of the municipality of Tehran.

To recruit the sample, Tehran was divided to four regions (North, South, East and West) and from each region, two citizen clubs were then selected randomly. Random sampling was then applied in each of the selected Tehran citizen clubs. The clubs were run by the private sector with the collaboration of Tehran municipality to provide free social services to older people with the goal of improving social, spiritual, psychological and health-related well-being. Sampling was performed from February 28, 2015 to September 2, 2015. The inclusion criteria were age $\geq 60$ years (start of old age in developing countries), willingness to take part in the study, ability to read and write in Farsi, no severe visual or hearing impairments and no history of treatment for psychiatric disorders (based on a score of $\geq 7$ on the Short-Mental State Examination using the Farsi version of the Abbreviated Mental Test [AMT] score). ${ }^{34}$

If a participant withdrew from the study, another qualified individual from a citizens' club was recruited. In accord with the literature on suggested sample size for factor analysis, 10-20 persons per item of the AAQ were selected. Therefore, the sample size goal in this study was 400 older people. The beginning of the assessment of psychometric properties of the AAQ was the process of the instrument's translation. After obtaining permission from the questionnaire's designer, the questionnaire was forward translated and adapted from English to Farsi by two translators and then back translated by two other translators without contact between them. The two translated versions were compared, and after slight modifications in wording, the final version was developed. This new version was again retranslated to English by another language expert who was unaware of the original questionnaire. The new and original English versions were compared to ensure that the concepts were transferred appropriately into the Farsi version. This version was presented to six experts in the field of Farsi language to revise it in terms of grammar, correct word usage and order of items. Next, the AAQ was validated using face, content and construct (exploratory factor analysis) validities. In addition, for reliability, its internal consistency and stability were assessed. Face validity was conducted by 15 older people. The content validity of the AAQ was assessed through the content validity index (CVI) and scale-level CVI/averaging calculation method (S-CVI/Ave) by six specialists (one geriatrician, one psychologist, three associate professors in nursing and one questionnaire development expert). According to the index proposed by Waltz and Bausell, CVI $\geq 0.79$ was considered acceptable. ${ }^{35,36}$

During the translation process, no changes were made to the number of items or the content of the original questionnaire. The items were assessed for compatibility with cultural aspects and differences. The desirable S-CVI/Ave was 0.90 as a higher score for the adoption of S-CVI/Ave was recommended. ${ }^{35}$

\section{Ethical approval}

The study protocol was approved by the ethics committee affiliated with Shahed University, Tehran, Iran (decree number: 932254812). The AAQ was translated with the written permission of Professor Laidlaw, the original developer of the AAQ. Official permission to recruit older people on site was obtained from the citizen clubs. The goals and procedures of the research were explained to the participants. They were ensured of confidentiality and that they could withdraw from the study at any time. Each participant signed a written informed consent form before filling out the instrument. The completed instruments were stored in a secure place.

\section{Data collection}

Data were collected from January to September 2015. The participants completed the instrument following instructions being presented by one of the researchers (MN). If a person was illiterate, an interview format was used to complete it. The average time required to complete the questionnaire was 18 minutes.

\section{Instruments}

Data were collected from participants using a demographic questionnaire, the Farsi version of the SF-36, the scale of WHOQOL-BREF, and the Farsi version of AAQ. ${ }^{37,38}$

\section{Demographic characteristics}

The demographic information questionnaire included questions about age, gender, marital status, residence, education level, economic status, employment status, history of smoking, history of illness and frequency of hospitalization in the last year.

\section{SF-36}

This was a general QoL instrument that measured the following eight health-related domains: physical functioning ( $\mathrm{PF}-10$ items), role limitations due to physical problems 
( RP - four items), bodily pain (BP - two items), general health perceptions ( $\mathrm{GH}$ - five items), vitality (VT - four items), social functioning ( $\mathrm{SF}$ - two items), role limitations due to emotional problems ( $\mathrm{RE}$ - three items) and perceived mental health ( $\mathrm{MH}$ - five items). It was scored from 0 to 100 with 0 indicting the worst and 100 the best situation. The validity of this instrument was confirmed, and its Cronbach's alpha coefficient was reported as $0.65 .^{37}$

\section{WHOQOL-BREF}

The Farsi version of the WHOQOL-BREF is a generic QoL questionnaire that assesses QoL in older people (age $\geq 60$ years). It comprises 26 statements and utilizes a 5-point Likert scale format to record responses. A total of 24 statements cover the four domains of "physical", "psychological", "social relationships" and "environment" with two global questions about overall QoL and satisfaction with health. Scores ranged 4-20 for each domain and 24-120 for the total score with higher scores representing a higher QoL related to the last 2 weeks. ${ }^{33}$ Reliability and validity of the original and the Farsi version of this questionnaire were confirmed. ${ }^{39}$

\section{Attitudes to Aging Questionnaire (AAQ)}

The AAQ was originally developed by Laidlaw et $\mathrm{a}^{27}$ and measures the subjective understanding and attitudes of older adults toward their own aging process across cultures. It contains 24 statements and explores the three dimensions of physical changes, psychological growth and psychosocial loss. Older people are asked to determine the degree of their agreement with each item on a 5-point Likert scale from 1 (strongly disagree or not at all true) to 5 (strongly agree or extremely true). Scores ranged from 8 to 40 for each dimension. The structure of the AAQ enables the capture of attitudes, both positive and negative, toward aging. The domain of psychological growth has an explicitly positive focus and could be summarized as "wisdom" or "growth" as it recognizes a lifespan development perspective on aging as viewed by the individual. Higher scores on physical changes and psychological growth dimensions demonstrate a more positive appraisal of one's own aging. In the domain of psychosocial losses, the perceptions and negative experiences of aging are collected. This domain enables negative attitudes and experiences to aging, as related to psychological and social losses, by older people to be measured. Higher scores on the psychosocial loss dimension display a more negative appraisal of aging. The total score is based on the total of 24 statements of the questionnaire and the summation of inverted scores for the psychosocial loss dimension. Higher scores reflect more positive attitudes toward one's own aging process. The AAQ uses two different formats so as to gain an experiential and a general approach to perceptions and individual attitudes. Section one contains individual experiential items, such as "growing older has been easier than I thought" and section two explores more general attitudes, such as "wisdom comes with age". This approach contributes to the potential efficiency of this questionnaire as it was developed to be used by policy makers, clinicians and researchers.

\section{Statistical analysis}

Descriptive and inferential statistics were used for data analysis via the software SPSS v.21 (IBM Corporation, Armonk, NY, USA). A $p$-value $<0.05$ was considered as statistically significant. Parametric statistics were computed where applicable, such as for variables with a normal distribution. Since the findings of parametric and nonparametric analyses were not different, the findings of nonparametric tests are presented in this article.

Convergent validity was assessed using the Pearson correlation test. Internal consistency for each domain and for the overall scale was assessed using the calculation of the Cronbach's alpha coefficient. An alpha of at least 0.70 was required to achieve an acceptable level of internal consistency. ${ }^{40}$ For evaluating construct validity and examining the factor structure of data, the varimax rotation with the Kaiser-Meyer-Olkin (KMO) index and the Bartlett test of sphericity were conducted. They tested if there was an underlying structure to the data, and $\mathrm{KMO}>0.40$ was considered to be acceptable. A significant Bartlett's sphericity test was statistically significant at the level of 0.0001 . This justified the use of factor analysis according to the correlation matrix that resulted in the study sample. Principal component analysis with a direct oblique rotation was conducted for the construct validity analysis. We preferred this oblique rotation to an orthogonal rotation (ie, varimax rotation); hence, Laidlaw et $\mathrm{al}^{27}$ indicated a higher order factor in their confirmatory factor analyses. It expected that variables represented a larger general concept (eg, attitudes toward own aging) and could be interrelated. The loading factors $\geq 0.30$ were determined as a criterion to define a salient factor. Every statement with the highest load on each of the three factors was considered as the statement related to that factor, which was not different from the original questionnaire. The number of factors retained was decided using the Kaiser's criterion of retaining factors with unrotated eigenvalues of $\sim>1.0$ and the scree plot. 
Acceptability was determined by using the AAQ missing values, summary score distributions, floor and ceiling effects as well as skewness and kurtosis statistics. Floor and ceiling effects were present, if $>10 \%$ of respondents achieved the lowest or highest possible summary score. ${ }^{41}$ Evidence of skewness or kurtosis was considered demonstrated if the absolute value of the statistic was higher than twice the standard error of the corresponding statistic.

Reliability was evaluated through measuring the level of internal consistency. Therefore, the Cronbach's alpha coefficient was calculated for the whole questionnaire and each dimension.

To investigate stability, the test-retest method was used after a 2-week interval and the cooperation of 80 older people. The scores were used to calculate the intraclass correlation coefficient (ICC). The known-group validity of the AAQ was evaluated by the subgroups of gender and educational status using the independent $t$-test. To assess convergent validity, the correlations between the total score of the AAQ and SF-36 and WHOQOL-BREF's scores for the QoL were calculated.

\section{Results}

\section{Sociodemographic characteristics of the samples}

Of the total sample population, $218(54.5 \%)$ were female and $286(71.5 \%)$ were married. The education level of most $(37.5 \%)$ was primary school. The mean \pm SD of their age was $67.39 \pm 6.14$ years, and $299(74.8 \%)$ participants were in the age group of $60-70$ years. In addition, $46.7 \%$ were pensioners and $55.5 \%$ had two to four children. The majority of participants were living with their spouses 283 (70.8\%) and had a low income (47.3\%; Table 1).

\section{Factor structure}

The KMO was $>0.40(\mathrm{KMO}=0.904)$, which was higher than the recommended value, ie, 0.60 , and the Bartlett test of sphericity was statistically significant $(p<0.001)$. It suggested the factorability of the correlation matrix, indicating an underlying structure in the instrument.

The S-CVI/Ave was reported as 0.92 . Three factors were extracted and identified using a minimum eigenvalue of 1.0 as the factor criterion. The visual inspection of the scree plot strongly supported the suggested three factor solutions accounting for $76.87 \%$ of the total variance (Figure 1).

Factor analysis was inspected following oblique rotation. The three-factor solution produced loading $\geq 0.40$ on this factor. The first factor corresponded to physical changes,
Table I Demographic and clinical data of the samples

\begin{tabular}{lll}
\hline Variables & Categories & $\mathbf{n}(\%)$ \\
\hline Gender & Female & $218(54.5)$ \\
Age group (years) & Male & $182(45.5)$ \\
& $60-70$ & $299(74.8)$ \\
Living arrangement & $71-80$ & $89(22.3)$ \\
& $8 I-90$ & $12(3.0)$ \\
& Alone & $65(16.3)$ \\
& Spouse & $283(70.8)$ \\
Education level & Children & $50(12.5)$ \\
& Others & $2(0.5)$ \\
& Illiterate & $66(16.5)$ \\
& Able to read and write & $40(10.0)$ \\
& Primary school & $150(37.5)$ \\
Marital status & Secondary school & $97(24.3)$ \\
& Academic degree & $47(11.8)$ \\
& Single & $4(1)$ \\
& Married & $286(71.5)$ \\
Employment status & Widow & $106(26.5)$ \\
& Divorced & $4(1)$ \\
& Housewife & $177(44.25)$ \\
& Self-employment & $21(5.25)$ \\
Current disease & Pensioner & $187(46.75)$ \\
& Out of work & $15(3.75)$ \\
& Yes & $357(89.25)$ \\
& No & $43(10.75)$ \\
\hline
\end{tabular}

the second factor was psychological growth and the third factor was psychosocial loss.

In the pattern matrix produced by the three-factor solution (Table 2), all items loaded $\geq 0.40$. There were no missing values for any of the subscales. A normal distribution was observed for all dimensions. No noteworthy floor or ceiling effects were found per subscale or for the total score.

\section{Internal consistency}

The Cronbach's alpha coefficient was reported as 0.90 for the total score, 0.85 for physical changes, 0.91 for psychological growth and 0.93 for psychosocial loss (Table 3). In addition, we noted that corrected item-total correlations were all above the threshold of 0.20 .

\section{Test-retest reliability}

Reported correlations were high for the total score and all dimensions $(r=0.32$ to $r=0.76 ; p<0.001)$. No statistically significant differences were reported between test and retest scores.

The ICC for the total instrument was reported as 0.74-0.88 (Table 3).

\section{Convergent validity}

Correlations between the AAQ and other questionnaires are reported in Tables 4 and 5. Strong positive correlations 


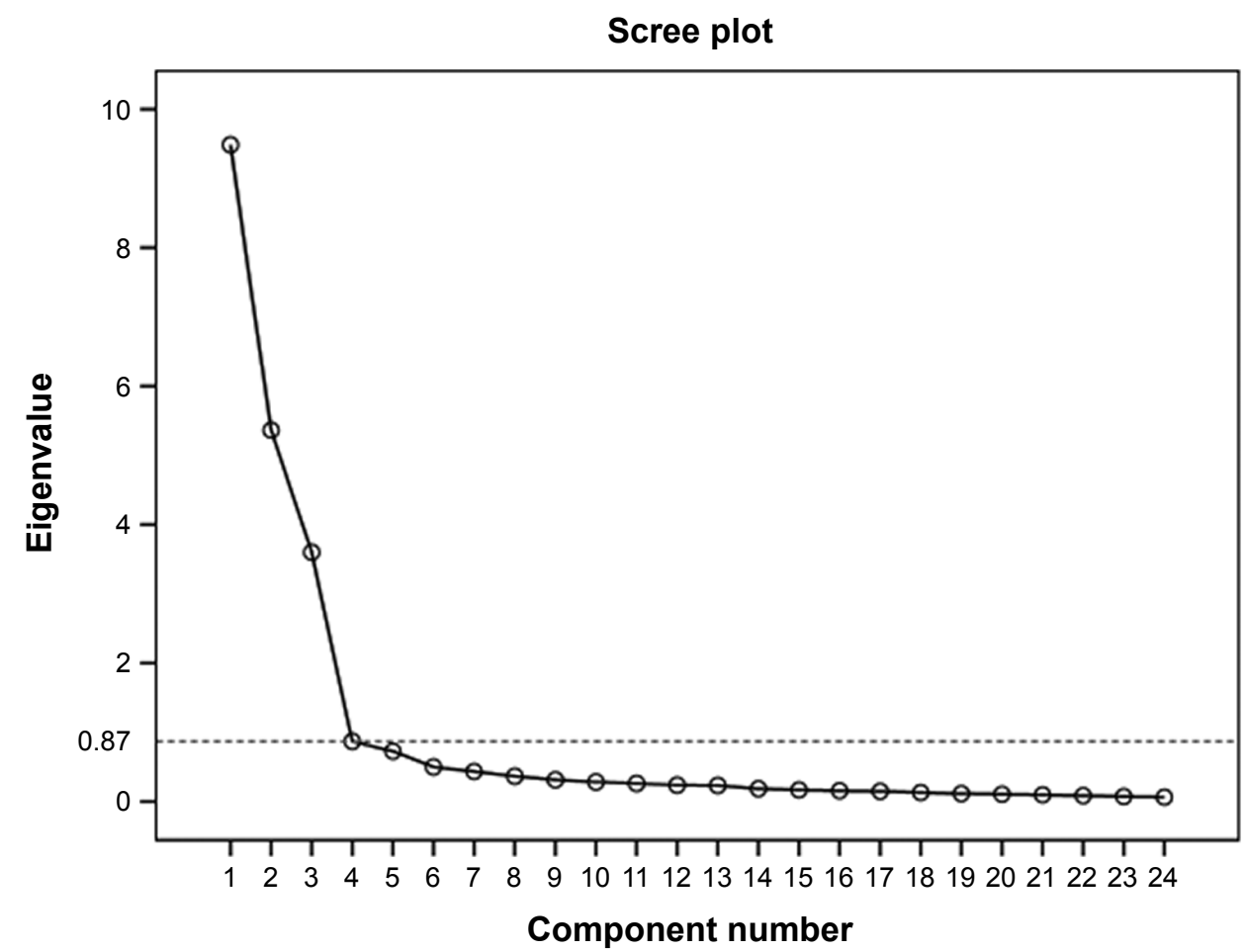

Figure I The scree plot for the samples $(n=400)$.

Table 2 Factor loading of the AAQ items

\begin{tabular}{|c|c|c|c|}
\hline \multirow[t]{2}{*}{ Items } & \multicolumn{3}{|l|}{ Factors } \\
\hline & $\begin{array}{l}\text { Physical } \\
\text { change }\end{array}$ & $\begin{array}{l}\text { Psychological } \\
\text { growth }\end{array}$ & $\begin{array}{l}\text { Psychosocial } \\
\text { loss }\end{array}$ \\
\hline 3. Old age is the time of loneliness & 0.85 & & \\
\hline 6. Old age is the time of depression & 0.88 & & \\
\hline 9. In old age it is more difficult to talk about feelings & 0.79 & & \\
\hline 12. Old age is the time of losing many things & 0.89 & & \\
\hline 15. In old age I have lost my ability and physical independence & 0.89 & & \\
\hline 17. In old age it is more difficult to find new friends & 0.88 & & \\
\hline 20. Now that I am older, I don't feel involved in the society & 0.89 & & \\
\hline 22. I feel excluded from things, because of my age & 0.91 & & \\
\hline I. As people get older, they are more able to cope with life & & 0.87 & \\
\hline 2. It is a privilege to grow old & & 0.85 & \\
\hline 4. In old age people become more reasonable and wise & & 0.89 & \\
\hline 5. There are many pleasant things about growing older & & 0.86 & \\
\hline 10. I am more accepting myself as I have grown older & & 0.85 & \\
\hline 18. It is important for me to share my life experiences with younger people & & 0.87 & \\
\hline 19. I believe that my life has made a difference & & 0.76 & \\
\hline 21. I like to be a good example for younger people & & 0.88 & \\
\hline 7. It is important to do exercise at any age & & & 0.61 \\
\hline 8. Growing older is easier than what I have thought & & & 0.88 \\
\hline II. I do not feel that I am old & & & 0.87 \\
\hline 13. My identity is not defined by my age & & & 0.88 \\
\hline 14. I have more energy now than I expected for my age & & & 0.83 \\
\hline 16. My physical problems in old age do not hinder me to reach my aims & & & 0.84 \\
\hline 23. My health is much better than what I have expected at my age & & & 0.90 \\
\hline 24. I keep myself as fit and active as possible through exercising & & & 0.77 \\
\hline Eigenvalues & 9.45 & 5.36 & 3.60 \\
\hline Explained variance & 27.29 & 25.72 & 23.88 \\
\hline
\end{tabular}

Abbreviation: AAQ, Attitudes to Aging Questionnaire. 
Table 3 The stability, internal consistency, reliability and ICC of the Farsi version of the AAQ

\begin{tabular}{|c|c|c|c|c|c|c|c|c|c|c|c|}
\hline \multirow[t]{2}{*}{ Dimensions } & \multirow[t]{2}{*}{$\begin{array}{l}\text { Number } \\
\text { of items }\end{array}$} & \multirow[t]{2}{*}{$\begin{array}{l}\text { Mean score } \\
\text { (SD), } n=80\end{array}$} & \multirow[t]{2}{*}{$\begin{array}{l}\text { Cronbach's } \\
\text { alpha coefficient }\end{array}$} & \multirow[t]{2}{*}{ ICC } & \multicolumn{2}{|c|}{$\mathrm{Cl}=0.95$} & \multirow[t]{2}{*}{$p$-value } & \multicolumn{4}{|c|}{$\begin{array}{l}\text { Pearson correlation } \\
\text { coefficient }\end{array}$} \\
\hline & & & & & Min & Max & & PC & PG & PL & Total \\
\hline PC & 8 & $20.17(5.22)$ & 0.85 & 0.74 & 0.62 & 0.82 & 0.000 & 1 & & & \\
\hline PG & 8 & $28.40(4.94)$ & 0.91 & 0.84 & 0.77 & 0.90 & 0.000 & 0.64 & I & & \\
\hline PL & 8 & $37.30(4.75)$ & 0.93 & 0.87 & 0.81 & 0.91 & 0.000 & 0.38 & 0.54 & I & \\
\hline Total & 24 & $80.65(10.72)$ & 0.90 & 0.82 & 0.74 & 0.88 & 0.000 & 0.76 & 0.32 & 0.55 & I \\
\hline
\end{tabular}

Abbreviations: ICC, intraclass correlation coefficient; AAQ, Attitudes to Aging Questionnaire; Min, minimum; Max, maximum; PC, physical change; PG, psychological growth; PL, psychosocial loss.

$(r=0.7, r=0.5-0.7)$ were reported between the total score of the AAQ and the WHOQOL-BREF and SF-36, respectively (Tables 4 and 5).

As expected, the more the people had a positive opinion of their physical functioning (physical changes), the less they reported the negative impacts of sensory losses on their QoL $(r=0.56, p<0.001 ; r=0.78, p<0.001)$.

\section{Discriminant validity}

No differences between male and female were reported for the total score and the three AAQ's dimensions (all $p>0.05$ ). Discriminant validity showed that male older people scored significantly higher on almost all aspects of the domains compared to female older people. Those participants with higher educational levels scored significantly higher on

Table 4 Correlation coefficients and the correlation between the Farsi version of the AAQ and SF-36 questionnaire $(n=400)$

\begin{tabular}{|c|c|c|c|c|}
\hline \multirow[t]{2}{*}{ SF-36 } & \multicolumn{4}{|l|}{ AAQ } \\
\hline & $\begin{array}{l}\text { Physical } \\
\text { change }\end{array}$ & $\begin{array}{l}\text { Psychological } \\
\text { growth }\end{array}$ & $\begin{array}{l}\text { Psychosocial } \\
\text { loss }\end{array}$ & Total \\
\hline \multirow[t]{2}{*}{ Physical functioning } & $0.73 * *$ & $0.67^{*}$ & $0.40 * *$ & $0.63 I^{* *}$ \\
\hline & 0.000 & 0.000 & 0.000 & 0.007 \\
\hline Role limitations due & $0.44 * *$ & $0.46 *$ & $0.88^{*}$ & $0.5 \mathrm{II}$ ** \\
\hline to physical problems & 0.000 & 0.000 & 0.004 & 0.0027 \\
\hline \multirow[t]{2}{*}{ Bodily pain } & $0.83^{* *}$ & $0.84 *$ & $0.72^{*}$ & $0.7 I^{* *}$ \\
\hline & 0.000 & 0.000 & 0.044 & 0.002 \\
\hline \multirow[t]{2}{*}{ General health } & $0.84 * *$ & $0.65^{* *}$ & $0.65 * *$ & $0.74 * *$ \\
\hline & 0.000 & 0.000 & 0.000 & 0.000 \\
\hline \multirow[t]{2}{*}{ Vitality } & $0.64 * *$ & $0.68 * *$ & $0.42 * *$ & $0.61^{* *}$ \\
\hline & 0.000 & 0.000 & 0.000 & 0.000 \\
\hline \multirow[t]{2}{*}{ Social functioning } & $0.75^{* *}$ & $0.85^{* *}$ & $0.63^{*}$ & $0.78 * *$ \\
\hline & 0.000 & 0.017 & 0.045 & 0.007 \\
\hline Role limitations & $0.31 * *$ & $0.97^{* *}$ & $0.73 * *$ & $0.62 * *$ \\
\hline due to emotional & 0.000 & 0.002 & 0.013 & 0.004 \\
\hline \multicolumn{5}{|l|}{ problems } \\
\hline \multirow[t]{2}{*}{ Mental health } & $0.64 * *$ & $0.96 * *$ & $0.74 * *$ & $0.61 * *$ \\
\hline & 0.000 & 0.000 & 0.000 & 0.000 \\
\hline
\end{tabular}

Notes: *Correlation is significant at the 0.05 level. ${ }^{* *}$ Correlation is significant at the 0.01 level.

Abbreviations: AAQ, Attitudes to Aging Questionnaire; SF-36, Short Form 36. physical changes and lower on psychological growth and psychosocial loss.

\section{Known-group validity}

The known-group validity of the AAQ was assessed using the parameters of gender and education level (Table 6). Participants with higher educational levels scored higher on the total score $(p<0.001)$ and physical changes $(p=0.060)$ but lower on psychosocial loss $(p<0.010)$.

\section{Discussion}

This study aimed to assess the psychometric properties of the Farsi version of the AAQ in terms of internal consistency, construct and convergent and discriminant validities using a sample of Iranian older people. The Farsi version of the AAQ investigated attitudes toward the aging process as a personal experience and gave priority to the subjective experience of aging, rather than the objective indicators of physical or psychological health outcomes. No individuals with cognitive impairments were included in this study.

Table 5 Correlation coefficients and the correlation between the Farsi version of the AAQ and WHOQOL-BREF questionnaire $(n=400)$

\begin{tabular}{|c|c|c|c|c|}
\hline \multirow[t]{2}{*}{ QoL } & \multicolumn{4}{|l|}{ AAQ } \\
\hline & $\begin{array}{l}\text { Physical } \\
\text { change }\end{array}$ & $\begin{array}{l}\text { Psychological } \\
\text { growth }\end{array}$ & $\begin{array}{l}\text { Psychosocial } \\
\text { loss }\end{array}$ & Total \\
\hline \multirow[t]{2}{*}{ Physical health } & $0.65 * *$ & $0.64 *$ & $0.97 * *$ & $0.73 * *$ \\
\hline & 0.000 & 0.000 & 0.000 & 0.007 \\
\hline \multirow[t]{2}{*}{ Psychological } & $0.84 * *$ & $0.65^{*}$ & $0.54 *$ & $0.63^{* *}$ \\
\hline & 0.000 & 0.000 & 0.004 & 0.002 \\
\hline \multirow[t]{2}{*}{ Social relationships } & $0.75^{* *}$ & $0.84^{*}$ & $0.63^{*}$ & $0.77^{* *}$ \\
\hline & 0.000 & 0.000 & 0.044 & 0.002 \\
\hline \multirow[t]{2}{*}{ Environment } & $0.72 * *$ & $0.64 * *$ & $0.84^{* *}$ & $0.73^{* *}$ \\
\hline & 0.000 & 0.000 & 0.000 & 0.000 \\
\hline \multirow[t]{2}{*}{ Total } & $0.54 * *$ & $0.96 * *$ & $0.53^{* *}$ & $0.73^{* *}$ \\
\hline & 0.000 & 0.000 & 0.001 & 0.000 \\
\hline
\end{tabular}

Notes: *Correlation is significant at the 0.05 level. ${ }^{* *}$ Correlation is significant at the 0.01 level.

Abbreviations: AAQ, Attitudes to Aging Questionnaire; WHOQOL-BREF, World Health Organization quality of life - brief; QoL, quality of life. 
Table 6 Comparison of known groups: average rating of AAQ based on the gender and educational level

\begin{tabular}{llllllll}
\hline Sociodemographics & Categories & $\begin{array}{l}\text { Physical } \\
\text { change }\end{array}$ & $\begin{array}{l}\boldsymbol{t} \text {-test, df, } \\
\boldsymbol{p} \text {-value }\end{array}$ & $\begin{array}{l}\text { Psychological } \\
\text { growth }\end{array}$ & $\begin{array}{l}\boldsymbol{t} \text {-test, df, } \\
\boldsymbol{p} \text {-value }\end{array}$ & $\begin{array}{l}\text { Psychosocial } \\
\text { loss }\end{array}$ & $\begin{array}{l}\boldsymbol{t} \text {-test, df, } \\
\boldsymbol{p} \text {-value }\end{array}$ \\
\hline Gender & Male $(\mathrm{n}=182)$ & $21.44(\mathrm{I} .03)$ & -45.59, & $32.45(2.92)$ & 51.54, & $30.14(7.38)$ & 39.54, \\
& Female $(\mathrm{n}=218)$ & $15.73(1.43)$ & $398,0.001$ & $18.83(2.31)$ & $398,0.002$ & $18.41(2.34)$ & $398,0.001$ \\
\multirow{2}{*}{ Educational level } & Higher $(\mathrm{n}=144)$ & $19.23(3.08)$ & -3.44, & $24.34(7.05)$ & 2.97, & $23.97(7.32)$ & 3.22, \\
& Lower $(\mathrm{n}=256)$ & $18.14(3.07)$ & $398,0.001$ & $26.55(7.34)$ & $398,0.060$ & $26.44(7.48)$ & $398,0.010$ \\
\hline
\end{tabular}

Note: Data presented as mean (standard deviation) unless stated otherwise.

Abbreviation: AAQ, Attitudes to Aging Questionnaire.

Questionnaire completion either by participant or personal interview did not affect responses. Findings of this study indicate that the AAQ is a good self-reporting measure and could be used with large samples without requiring the presence of an interviewer (ie, online surveys). The AAQ had good test-retest reliability and internal consistency. Furthermore, the structure of the Farsi version of the AAQ was similar to that of the original one. Concerning the factor structure, the dimension of physical changes was less conceptually homogenous. In fact, the distribution of items demonstrated remarkable congruence with the factor solution reported by Laidlaw et al. Such results led us to choose the same structure as the original one with three factors. ${ }^{40}$

It is worth noting that each item and domain presented in this study were similar to those reported in the original version of Laidlaw et al. ${ }^{14,27}$ Similarly, Lucas-Carrasco et al ${ }^{28}$ in Spain, Chachamovich et $\mathrm{al}^{29}$ in Brazil and Kalfoss et $\mathrm{al}^{26}$ in Norway also used factor analysis to determine the construct validity of the AAQ.

Concerning acceptability, no floor or ceiling effects were observed for any of the dimensions or the total score. This quality is important; hence, floor and ceiling effects may limit a questionnaire's ability to reveal changes (lower or higher scores) over time. The internal consistency (Cronbach's $\alpha$ ) obtained for this questionnaire was acceptable in comparison to its English version. In this study, the Cronbach's alpha coefficient of the AAQ indicated a good internal consistency reliability, indicating that the reliability of the Farsi version of the AAQ was confirmed. Other studies reported the same results. ${ }^{26,28,42}$

With regard to the internal consistency of the AAQ, the Cronbach's alpha value of the total score $(\alpha=0.90)$ was similar to the score acquired in studies in Turkey $(\alpha=0.86),{ }^{15}$ Norway and Canada $(\alpha=0.86){ }^{26,42}$ These findings indicate that statements can be composed to form a summary score. Furthermore, the psychological growth dimension demonstrated the lowest reliability, as observed in other validation studies. ${ }^{28}$ It is possible that the statements of the AAQ are more sensitive to the translation procedure. Both an empirical and general approach to aging can lead to a variety of answers despite statements being related to the same dimension, for instance, participants might have various views on general versus empirical statements. In fact, they might respond to general statements by considering their perspectives about older adults in general. It is possible that older people may have a more negative opinion of aging, if they think about other older adults in general rather than their own experiences in life. The psychological growth dimension included four statements utilizing a general approach against two statements for the psychosocial loss dimension and one for the physical change dimension. Hence, the psychological growth dimension's outcomes might be interpreted with caution. In terms of convergent validity, strong positive correlations were found between the total score of the AAQ and the WHOQOL-BREF and SF-36, respectively (Tables 4 and 5). Some dimensions of these scales were also correlated with each other. Consistent with a previous validation study, moderate correlations were found between the AAQ dimensions and the WHOQOL. ${ }^{28}$ This emphasizes that those older adults who perceive their aging as a positive experience will experience also an enhanced QoL.

Consistent with other studies (Kalfoss et $\mathrm{al}^{26}$ and Top et $\mathrm{al}^{15,16}$ ), significant correlations are reported between most AAQ dimensions and WHOQOL facets. All these findings demonstrate associations between attitudes toward one's own aging and QoL in different cultures such as Norwegian, English Canadian, Spanish, Belgian and Turkish populations. ${ }^{15,16,26,28}$ Furthermore, a global study indicates that attitudes toward one's own aging assessed using the AAQ might mediate the relationship between QoL and satisfaction with health. ${ }^{43}$ This cross-sectional study of 4,593 older adults from different countries demonstrated that those people who were dissatisfied with their health had more negative attitudes toward their own aging process, which led them to experience a poorer QoL. Findings from this study indicate a positive correlation between positive attitudes toward one's own aging and good subjective physical health. These findings are congruent with a cross-sectional study that demonstrates that a negative opinion about aging collates with the individual's 
own physical health experience. However, it also emphasizes that current physical health may impact upon attitudes toward one's own aging and hence older adults' QoL. ${ }^{21}$ These findings highlight the significance of taking attitudes about one's own aging into account.

Consistent with the findings of other studies, differences between men and women on the AAQ dimensions and total score were reported. Finally, the AAQ indicated a good knowngroup validity. ${ }^{15,16}$

A Spanish study reports that higher educated people have a more positive opinion of their physical functioning and aging and less negative opinion of their psychosocial losses: this study also found this. Reasons for this finding may include the probability that higher educated people have more personal resources that can help them adjust to age-related issues and so maintain positive attitudes toward their own aging.

In terms of discriminant validity, associations between the AAQ and sociodemographic variables were reported. Older people's gender has been found to significantly shape attitudes toward the physical changes of aging in Brazil, but when looking across cultures, age hardly influences attitudes toward physical changes and women are more positive about psychological growth. ${ }^{29}$ Our findings are consistent with those of previous reports. Discriminant validity in the study of Lucas-Carrasco et $\mathrm{al}^{28}$ showed that male older people scored significantly higher on physical changes and lower on the psychosocial loss domain. In addition, Levy and Myers ${ }^{44}$ in the USA found that age and gender consistently influenced beliefs about aging. Women appear to be in a disadvantaged position as they get older, particularly when it comes to expectations of physical, mental and social decline. The effect of gender on the perceptions of bodily aging is less clear. ${ }^{45}$ It is suggested that subjective health appraisals can also be affected by gender. ${ }^{44}$ In the Spanish sample, the results showed good construct validity, and the AAQ results differed between groups at different levels of education. ${ }^{28}$ A strength of this study is that the stability of the AAQ questionnaire was also confirmed.

\section{Limitations}

As a limitation of this study, the literacy of the participants was $16.5 \%$ and the questionnaires were completed through interviews. The participants were selected from a group of relatively healthy and younger older people. In addition, there was a lack of a standard Farsi instrument for concurrent validity. The cross-sectional nature of this study prevents a determination of causality. Indeed, according to the current ideology in the Iranian society, older people are cared for in their own homes by their children and family members. Therefore, no older people living in care facilities were included in this study. However, the random sampling method and a relatively large sample size helped with the generalizability of the study findings. This study can be a starting point for other researchers to use the AAQ and compare differences between eastern and western contexts regarding attitudes to aging and older people care.

\section{Conclusion}

The AAQ is regarded as a suitable instrument to investigate older adults' attitudes toward their own aging. The Farsi version of the AAQ is a reliable, valid and consistent tool to assess attitudes toward aging and can be applied in similar cross-cultural contexts. In fact, the Farsi version of the AAQ has a similar structure to the original version. The presence of this instrument in Farsi can help health care providers working with older people, either in a research or clinical context, to investigate the effects of their interventions.

Since the tool's psychometric properties reflect a continuous and contiguous process, the next phase should be to confirm the confirmatory factor analysis structure of the AAQ with others samples from the same people. Prior to this tool being applied in other studies, confirmatory factor analyses should also be undertaken to evaluate variances across age groups. It is further suggested that to examine the psychometric properties of the AAQ in various populations of older adults would be useful. A strength of the AAQ is that it is an accessible questionnaire with features such as simple scoring, acceptable reliability and validity and can be completed in a short time and be applied in relatively different settings and contexts.

In line with longitudinal studies conducted by Levy et al, ${ }^{9}$ use of this instrument can add to the existing literature by its predictive power and ability to express the relationship between attitudes toward one's own aging and health-related consequences among Farsi-speaking people.

The use of this instrument by gerontologists, nurses, caregivers, health care educators and researchers can improve their knowledge and understanding of aging and improve QoL among older people, which can then enhance their satisfaction with care. Given the cognitive nature of attitudes toward aging, further qualitative studies in this area are required to identify other possible dimensions. Moreover, interventions for improving social support in individuals with depression, functional and social limitations can be based on the information collected by using the AAQ. The collected information can be used by health care educators to inform 
future health care providers of the needs of older people and how to provide the most appropriate care for them.

\section{Acknowledgments}

We would like to thank the older people who participated in this study. The authors are grateful to Professor K Laidlaw for granting the permission to translate and use the original version of the AAQ.

\section{Author contributions}

NR participated in the study design, data collection, statistical analysis and drafting of the manuscript. MHK contributed toward study design and statistical analysis. MV and PG were responsible for drafting the manuscript. $\mathrm{MN}$ and $\mathrm{TB}$ were involved in data collection. All authors approved the final version to be published. All authors contributed toward data analysis, drafting and critically revising the paper and agree to be accountable for all aspects of the work.

\section{Disclosure}

The authors report no conflicts of interest in this work.

\section{References}

1. Sadegh Moghadam L, Foroughan M, Mohammadi F, Ahmadi F, Farhadi A. Validity and reliability of the Farsi version of the Brief Aging Perceptions Questionnaire in Iranian older adult Clinical Interventions in Aging. Clin Interv Aging. 2016;11:507-511.

2. Karami Matin B, Rezaei S, Shaahmadi F, Kazemi Karyani A. [Ageing in Iran in 1410, a warning to health care system]. Teb va Tazkieh. 2012;22(2):9-18.

3. Sheykhi M. Social security and the Elderly People's pathology in Tehran: a sociological study. Salmand Iran J Ageing. 2008;2:454-461.

4. de Freitas MC, Queiroz TA, de Sousa JA. The meaning of old age and the aging experience of in the elderly. Rev Esc Enferm USP. 2010;44(2): 407-412.

5. Demakakos P, Hacker E, Gjonça E. Perceptions of ageing. In: Banks J, Breeze E, Lessof C, Nazroo J, editors. Retirement, Health and Relationships of the Older Population in England: The 2004 English Longitudinal Study of Ageing (Wave 2). London: The Institute for Fiscal Studies; 2006:339-351.

6. Cuddy AJ, Fiske ST, Kwan VS, et al. Stereotype content model across cultures: towards universal similarities and some differences. Br J Soc Psychol. 2009;48(1):1-33.

7. Dimakakos P, Hacker E, Gjonca E. Perceptions of ageing. In: Banks J, Breeze E, Lessof C, Nazroo J, editors. In Retirement, Health and Relationships of the Older Population in England: The 2004 English Longitudinal Study of Ageing (Wave 2). London: The Institute for Fiscal Studies; 2006:339-366.

8. Levy BR, Zonderman AB, Slade D, Ferrucci L. Memory shaped by age stereotypes over time. J Gerontol B Psychol Sci Soc Sci. 2012;67(4): 432-436.

9. Levy BR, Zonderman AB, Slade MD, Ferrucci L. Age stereotypes held earlier in life predict cardiovascular events in later life. Psychol Sci. 2009;20(3):296-298.

10. Sexton E, King-Kallimanis BL, Morgan K, McGee H. Development of the brief ageing perceptions questionnaire (B-APQ): a confirmatory factor analysis approach to item reduction. BMC Geriatr. 2014; 14(1):44.
11. Hess TM. Attitudes toward aging and their effects on behavior. In: Birren JE, Schaie KW, editors. Handbook of the Psychology of Aging. 6th ed. San Diego, CA: Academic Press; 2006:379-406.

12. Harrison T, Blozis S, Stuifbergen A. Longitudinal predictors of attitudes toward ageing among women with multiple sclerosis. Psychol Ageing. 2006;23(4):823-832.

13. Barker M, O'Hanlon A, McGee HM, Hickey A, Conroy RM. Crosssectional validation of the Aging Perceptions Questionnaire: a multidimensional instrument for assessing self-perceptions of aging. $B M C$ Geriatr. 2007;7:9.

14. Laidlaw K, Power M, Schmidt S. The Attitudes to Ageing Questionnaire (AAQ): development and psychometric properties. Int $J$ Geriatr Psychiatry. 2007;22(4):367-379.

15. Top M, Eris H, Kabalcioglu F. Quality of life (QOL) and attitudes toward aging in older adults in Sanliurfa, Turkey. Res Aging. 2012;35(5): $533-562$.

16. Top M, Eris H, Kabalcioglu F. Quality of life and attitudes toward aging among older women in Turkey. Affilia. 2012;27(4):406-419.

17. Löckenhoff CE, De Fruyt F, Terracciano A, et al. Perceptions of aging across 26 cultures and their culture-level associates. Psychol Aging. 2009;24(4):941-954.

18. Musaiger AO, D'Souza R. Role of age and gender in the perception of aging: a community-based survey in Kuwait. Arch Gerontol Geriatr. 2009;48(1):50-57.

19. Yun RJ, Lachman ME. Perceptions of aging in two cultures: Korean and American views on old age. J Cross Cult Gerontol. 2006;21(1-2): $55-70$.

20. Ostir GV, Ottenbacher KJ, Markides KS. Onset of frailty in older adults and the protective role of positive affect. Psychol Aging. 2004;19(3): 402-408.

21. Levy BR, Slade MD, Kasl SV. Longitudinal benefit of positive selfperceptions of aging on functional health. J Gerontol B Psychol Sci Soc Sci. 2002;57(5):409-417.

22. Levy BR, Slade MD, Kunkel SR, Kasl SV. Longevity increased by positive self-perceptions of aging. J Pers Soc Psychol. 2002;83(2):261-270.

23. Levy BR, Myers LM. Preventive health behaviors influenced by selfperceptions of aging. Prev Med. 2004;39(3):625-629.

24. Diehl M, Wahl HW, Barrett AE, et al. Awareness of aging: theoretical considerations on an emerging concept. Dev Rev. 2014;34(2):93-113.

25. Brothers A, Miche M, Wahl HW, Diehl M. Examination of associations among three distinct subjective aging constructs and their relevance for predicting developmental correlates. J Gerontol B Psychol Sci Soc Sci. Epub 2015 Oct 1:1-15.

26. Kalfoss MH, Low G, Molzahn AE. Reliability and validity of the attitudes to ageing questionnaire for Canadian and Norwegian older adults. Scand J Caring Sci. 2010;24:75-85.

27. Laidlaw K, Power M, Schmidt S; WHOQOL-OLD Group. The Attitudes to Ageing Questionnaire (AAQ): development and psychometric properties. Int J Geriatr Psychiatry. 2007;22(4):367-379.

28. Lucas-Carrasco R, Laidlaw K, Gomez-Benito J, Power MJ. The suitability of the Attitudes Ageing Questionnaire (AAQ) for Spanish older adults. Int Psychogeriatr. 2013;25(3):490-499.

29. Chachamovich E, Fleck M, Laidlaw K, Power M. Impact of major depression and subsyndromal symptoms on quality of life and attitudes toward ageing in an international sample of older adults. Gerontologist. 2008;48:593-602.

30. Shenkin SD, Watson R, Laidlaw K, Starr JM, Deary IJ. The attitudes to the Attitudes to Ageing Questionnaire: Mokken Scaling Analysis. PLoS One. 2014;9(6):e99100.

31. Power M, Quinn K, Schmidt S; WHOQOL-OLD Group. Development of WHOQOL-OLD module. Qual Life Res. 2005;14:2197-2214.

32. The WHOQOL Group. The World Health Organization quality of life assessment (WHOQOL): development and general psychometric properties. Soc Sci Med. 1998;46(12):1569-1585.

33. The WHOQOL Group. Development of the World Health Organization WHOQOL-BREF quality of life assessment. Psychol Med. 1998;28(3): $551-558$. 
34. Bakhtiari F, Foroughan M, Fakhrzadeh H, et al. Validation of the Farsi version of Abbreviated Mental Test (AMT) in elderly residents of Kahrizak Charity Foundation. Iran J Diabetes Metab. 2014;13(6): 487-494.

35. Polit DF, Beck CT. The content validity index: are you sure you know what's being reported? Critique and recommendations. Res Nurs Health. 2006;29(5):489-497.

36. Polit DF, Beck CT, Owen SV. Is the CVI an acceptable indicator of content validity? Appraisal and recommendations. Res Nurs Health. 2007;30(4):459-467.

37. Montazeri A, Goshtasebi A, Vahdaninia M, Gandek B. The short form health survey (SF-36): translation and validation study of the Iranian version. Qual Life Res. 2005;14(3):875-882.

38. Yousefy AR, Ghassemi GR, Sarrafzadegan N, Mallik S, Baghaei AM, Rabiei K. Psychometric properties of the WHOQOL-BREF in an Iranian adult sample. Community Ment Health J. 2010;46:139-147.

39. Nedjat S, Montazeri A, Holakouie K, Mohammad K, Majdzadeh R. Psychometric properties of the Iranian interview-administered version of the World Health Organization's Quality of Life Questionnaire (WHOQOL-BREF): a population-based study. BMC Health Serv Res. 2008;8:61.
40. Waltz CF, Strickland OL, Lenz ER. Measurement in Nursing and Health Research. 3rd ed. New York, NY: Springer; 2005.

41. Perales J, Cosco TD, Stephan B, Haro JM, Brayne C. Health-related quality-of-life instruments for Alzheimer's disease and mixed dementia. Int Psychogeriatr. 2013;25(5):691-706.

42. Eser E, Gerceklioglu GS, Eser S, et al. Psychometric properties of the Turkish version (AYTA-Tr) of the Who-Europe Attitudes of Aging (EAAQ) Questionnaire. Turk J Geriatr J. 2011;14:101-110.

43. Low G, Molzahn AE, Schopflocher D. Attitudes to aging mediate the relationship between older. Health Qual Life Outcomes. 2013; 28(11): 146 .

44. Levy BR, Myers LM. Relationship between respiratory mortality and self-perceptions of aging. Psychol Health. 2005;20(5):553-564.

45. Baker L, Gringart E. Body image and self-esteem in older adulthood ageing. Ageing Soc. 2009;29:977-995.
Clinical Interventions in Aging

\section{Publish your work in this journal}

Clinical Interventions in Aging is an international, peer-reviewed journal focusing on evidence-based reports on the value or lack thereof of treatments intended to prevent or delay the onset of maladaptive correlates of aging in human beings. This journal is indexed on PubMed Central, MedLine,

\section{Dovepress}

CAS, Scopus and the Elsevier Bibliographic databases. The manuscript management system is completely online and includes a very quick and fair peer-review system, which is all easy to use. Visit http://www.dovepress. com/testimonials.php to read real quotes from published authors. 